\title{
Decreased glutathione biosynthesis contributes to EGFR T790M-driven erlotinib resistance in non-small cell lung cancer
}

Hongde $\mathrm{Li}^{1,2,9}$, William Stokes ${ }^{3,9}$, Emily Chater ${ }^{3,9}$, Rajat Roy ${ }^{3,9}$, Elza de Bruin ${ }^{4,5}$, Yili Hu², Zhigang Liu ${ }^{2}$, Egbert F Smit ${ }^{6}$, Guus JJE Heynen ${ }^{7}$, Julian Downward ${ }^{4}$, Michael J Seckl ${ }^{3}$, Yulan Wang ${ }^{2,8}$, Huiru Tang ${ }^{1,2}$, Olivier E Pardo ${ }^{3}$

${ }^{1}$ State Key Laboratory of Genetic Engineering, Ministry of Education Key Laboratory of Contemporary Anthropology, Collaborative Innovation Centre for Genetics and Development, Shanghai International Centre for Molecular Phenomics, Metabonomics and Systems Biology Laboratory, Department of Biochemistry, School of Life Sciences, Zhongshan Hospital, Fudan University, Shanghai, China; ${ }^{2}$ Key Laboratory of Magnetic Resonance in Biological Systems, National Centre for Magnetic Resonance in Wuhan, State Key Laboratory of Magnetic Resonance and Atomic and Molecular Physics, Wuhan Institute of Physics and Mathematics, Chinese Academy of Sciences, Wuhan, China; ${ }^{3}$ Division of Cancer, Department of Surgery and Cancer, Imperial College, Hammersmith Hospital, London, UK; ${ }^{4}$ Signal Transduction Laboratory, CRUK London Research Institute, London, UK; ${ }^{5}$ Personalised Healthcare \& Biomarkers, Innovative Medicines and Early Development Biotech Unit, AstraZeneca, Cambridge, UK; ${ }^{6}$ Department of Pulmonary Diseases, VU University Medical Centre, Netherlands Cancer Institute, Amsterdam, Netherlands; ${ }^{7}$ Section of Molecular Carcinogenesis, Netherlands Cancer Institute, Amsterdam, Netherlands; ${ }^{8}$ Collaborative Innovation Centre for Diagnosis and Treatment of Infectious Diseases, Hangzhou, China

Epidermal growth factor receptor (EGFR) inhibitors such as erlotinib are novel effective agents in the treatment of EGFRdriven lung cancer, but their clinical impact is often impaired by acquired drug resistance through the secondary T790M EGFR mutation. To overcome this problem, we analysed the metabonomic differences between two independent pairs of erlotinib-sensitive/resistant cells and discovered that glutathione (GSH) levels were significantly reduced in T790M EGFR cells. We also found that increasing GSH levels in erlotinib-resistant cells re-sensitised them, whereas reducing GSH levels in erlotinib-sensitive cells made them resistant. Decreased transcription of the GSH-synthesising enzymes (GCLC and GSS) due to the inhibition of NRF2 was responsible for low GSH levels in resistant cells that was directly linked to the T790M mutation. T790M EGFR clinical samples also showed decreased expression of these key enzymes; increasing intra-tumoural GSH levels with a small-molecule GST inhibitor re-sensitised resistant tumours to erlotinib in mice. Thus, we identified a new resistance pathway controlled by EGFR T790M and a therapeutic strategy to tackle this problem in the clinic.

Keywords: metabolomics; glutathione; lung cancer; drug resistance; TKI

Cell Discovery (2016) 2, 16031; doi:10.1038/celldisc.2016.31; published online 27 September 2016

\footnotetext{
${ }^{9}$ These authors contributed equally to this work.

Correspondence: Michael J Seckl

Tel: +442033111421;

E-mail: m.seckl@imperial.ac.uk

or Huiru Tang

Tel: +86-21-51630725;

E-mail: huiru_tang@fudan.edu.cn

or Olivier E Pardo

Tel: +442075942814;

E-mail: o.pardo@imperial.ac.uk

Received 27 April 2016; accepted 14 July 2016
}

Introduction

Lung cancer is the main cancer killer and non-small cell lung cancer (NSCLC) represents $\sim 85 \%$ of such cases. About $10 \%$ and $30 \%$ of NSCLCs in Western and Asian populations, respectively, express an activated mutant epidermal growth factor receptor (EGFRm) and the vast majority $(90 \%)$ of such patients respond to ATP-competitive EGFR tyrosine kinase inhibitors (TKIs) such as gefitinib or erlotinib [1-3]. Unfortunately, most patients can quickly acquire TKI 
resistance limiting the benefits of these drugs to patients' survival.

Resistance mechanisms include Met amplification ( $5 \%$ of cases) [4] and more frequently (50\% of cases) an additional T790M gatekeeper mutation within the EGFRm [5-8]. The latter enhances kinase activity by increasing the affinity of EGFR for ATP, competing out TKI binding [9]. This led to the development of compounds irreversibly interacting with EGFRm/T790M, such as afatinib, 324674 and more recently AZD9291 and CO1696 [10-12]. However, thus far clinical trials of afatinib failed to demonstrate improved response in EGFRm/T790M patients [13], and although initial trials with the irreversible inhibitor AZD9291 showed great promises, additional resistance mechanisms to these inhibitors have already surfaced [14].

Changes in cellular metabolism accompany tumourigenesis and classical chemoresistance [15-17]. Hence, changes in metabolite concentrations can specifically reflect the onset of therapy resistance, providing response/outcome biomarkers and novel therapeutic strategies to reverse resistance [18, 19]. Both ${ }^{1} \mathrm{H}$-nuclear magnetic resonance (NMR) and mass spectrometry are efficient tools to investigate these metabolic changes [20-23].

Here we used ${ }^{1} \mathrm{H}-\mathrm{NMR}$ to compare the metabolic signatures of paired NSCLC cell lines expressing EGFRm without (erlotinib sensitive) or with the additional T790M mutation (erlotinib resistant). We showed that glutathione (GSH) levels were reduced in erlotinib-resistant NSCLC cells in a T790M-dependent manner due to the decreased expression of GSH-synthesising enzymes. Correcting this defect re-sensitised resistant cells to erlotinib in vitro and in vivo. Moreover, ethacrynic acid (EA), a glutathione$S$-transferase inhibitor, reversed erlotinib resistance in T790M NSCLC cells in vitro and in vivo by increasing GSH levels. As EA is a clinically used diuretic, it could be repurposed to reverse T790M-mediated erlotinib resistance in NSCLC patients. Overall, our work demonstrated the power of metabonomic screening to generate novel research hypotheses and discover unexplored strategies to tackle drug resistance in lung cancer treatments.

\section{Results}

\section{${ }^{1} H$-NMR-based metabolic profiling reveals decreased GSH levels in erlotinib-resistant NSCLC cells}

Two pairs of cell lines were employed to obtain generic metabonomic phenotypes for the erlotinibsensitive and erlotinib-resistant NSCLC cells. The first pair were the isogenically matched PC9 (erlotinib sensitive) and PC9ER (erlotinib resistant) cells both containing $\triangle$ E746-A750 EGFRm with an additional T790M (EGFRm/T790M) mutation in PC9ER cells. The second pair included the H3255 and genetically unrelated erlotinib-resistant H1975 cell lines sharing L858R EGFRm, but with an additional T790M mutation in the H1975. PC9ER and H1975 cells displayed significant resistance to erlotinib as compared with their sensitive counterparts (Supplementary Figure S1A). This resistance was limited to EGFR TKIs as PC9ER and PC9 cells were equally sensitive to conventional chemotherapeutic agents (Supplementary Figure S1B). It has been suggested that the EGFR T790M-mediated TKI resistance is due to increased affinity of the receptor for ATP, which displaces competitive inhibitors such as erlotinib [24]. However, both PC9ER and H1975 showed significant resistance even to the irreversible EGFR inhibitor 324674 compared with PC9 and H3255 cells, respectively (Supplementary Figure S1C). This clearly suggests that other unidentified molecular mechanisms also contribute to T790M-mediated TKI resistance.

To identify these, we comprehensively analysed the ${ }^{1} \mathrm{H}-\mathrm{NMR}$ metabonomic profiles of our erlotinibsensitive and -resistant cells. ${ }^{1} \mathrm{H}-\mathrm{NMR}$ analysis of cell extracts from our cell lines identified 36 metabolites (Figure 1a) for which unambiguous assignments were obtained using various two-dimensional NMR methods (Supplementary Table S1). Statistical analysis of the spectral data by orthogonal projections to latent structures discriminant analysis (OPLS-DA) showed significant metabonomic differences between the erlotinib-resistant and -sensitive cells (Figure $1 \mathrm{~b}$ and c). Changes in 14 metabolites mainly involved in GSH, amino acids, nucleotides and choline metabolism (Supplementary Figure S2A-C) correlated with resistance in both cell line pairs (Figure 1d; Supplementary Table S2). Noticeably, a significant drop in the intracellular levels of GSH accompanied erlotinib resistance (Figure 1d; Supplementary Table S2). Such GSH decrease observed by NMR was independently confirmed using a colorimetric assay (Figure 1e and f). This was intriguing, as drug resistance was traditionally associated with increased GSH levels [25, 26]. Nevertheless, GSH covalently binds some chemotherapeutic drugs leading to their glutathione$S$-transferase-mediated extracellular export and resistance of cancer cells to these compounds [27, 28]. Hence, the increased export of this metabolite in complex with erlotinib could account for the lower GSH levels in these resistant cell lines. ${ }^{1} \mathrm{H}-\mathrm{NMR}$ 

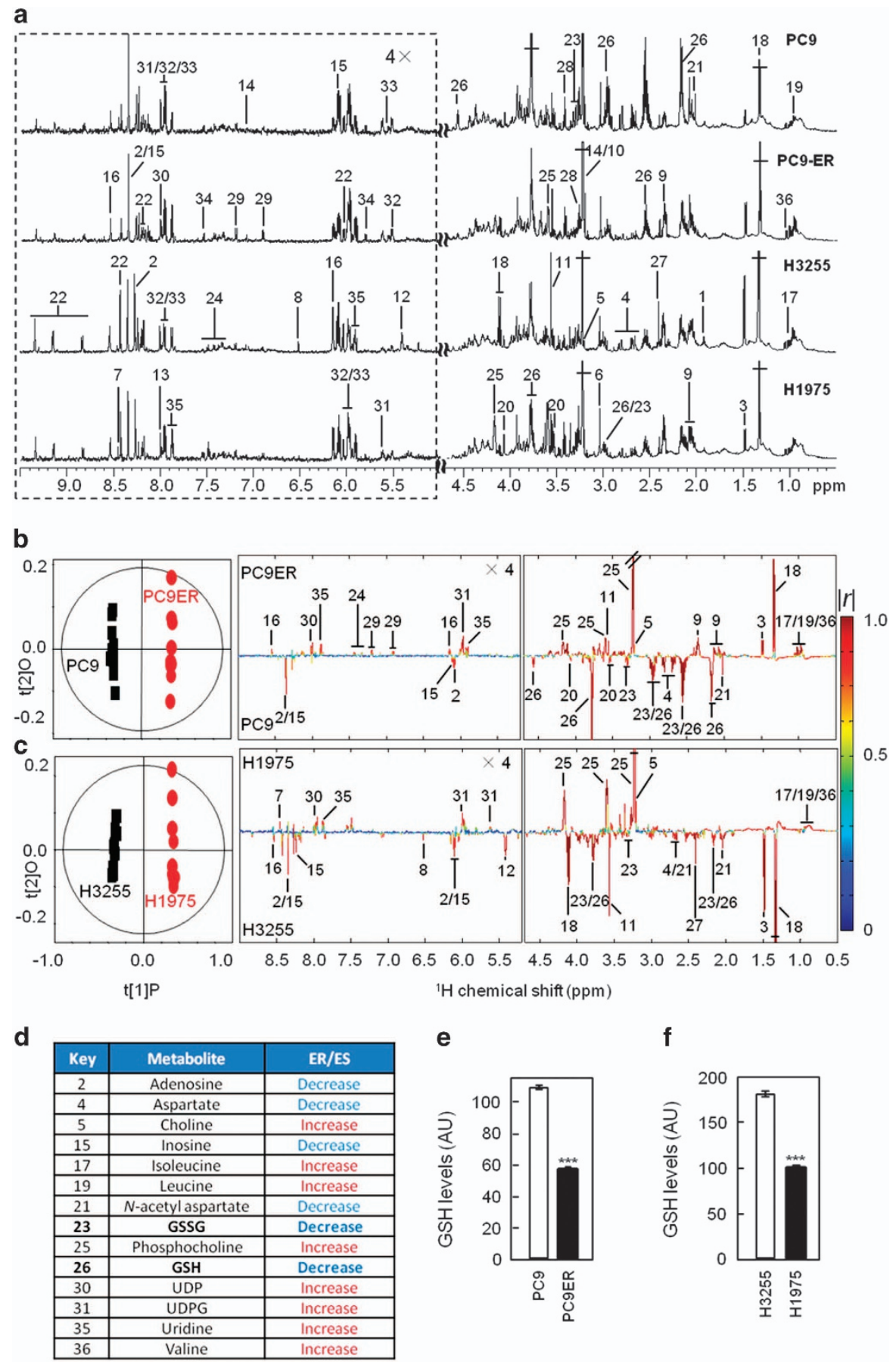

e
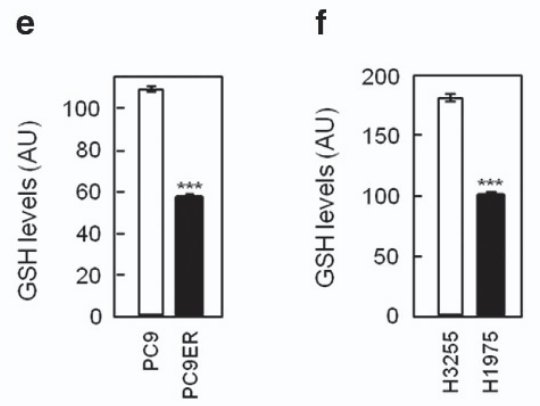

Figure 1 Metabolic characteristics for the erlotinib-resistant and -sensitive cells. (a) Typical $600 \mathrm{MHz}{ }^{1} \mathrm{H}-\mathrm{NMR}$ spectra of aqueous extracts from PC9, PC9ER, H3255 and H1975 cells. The region ( $\delta$ 5.0-9.5) is vertically expanded four times $(4 \times)$. Data representative of $n=10$. Orthogonal projections to latent structures discriminant analysis (OPLS-DA) score plots (left) and coefficient plots (right) for ${ }^{1} \mathrm{H}-\mathrm{NMR}$ spectra of aqueous cellular extracts from PC9ER and PC9 showing significantly differentiated metabolites (b), H1975 and H3255 (c). Models validated by CV-ANOVA, $P=2.36 \times 10^{-17}$ (b) and $P=3.04 \times 10^{-19}$ (c). The $Q^{2}$ is 0.99 for both models. The colour scale for coefficient plots reflects the differences in the contribution of metabolite variations between groups. In cutoff value is $0.602(n=10, P<0.05)$. For identification of peak numbers, see Supplementary Table S1 and d. (d) Metabolites showed statistically significant differences between resistant and sensitive cells in both cell line pairs with statistically significant 'decreases' or 'increases' detected in the erlotinib-resistant (ER) cells as compared with erlotinib-sensitive (ES) ones. (e, f) GSH levels in PC9 and PC9ER (e) or H3255 and H1975 (f) cells determined by colorimetric assay. Data are average \pm s.e.m. of $n=4$. Statistics: Student's $t$-test. ${ }^{* * *} P<0.001$. See also Supplementary Figures S1 and S2. 
a
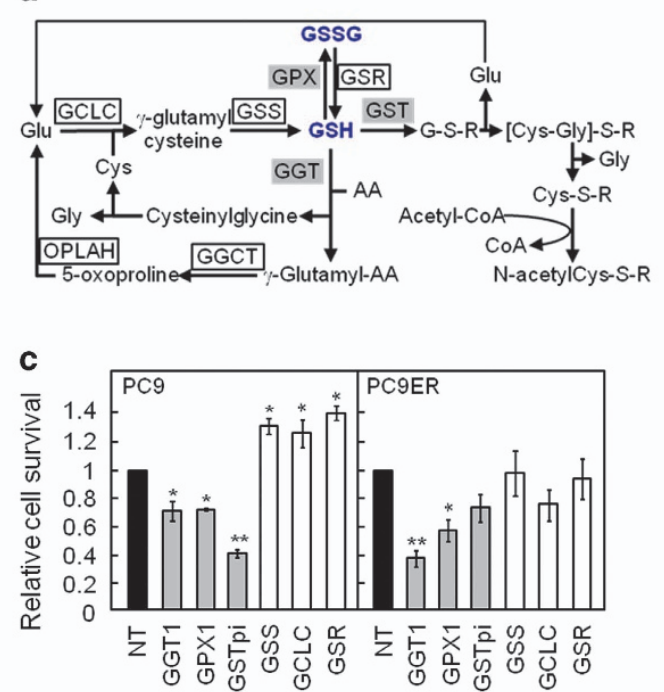

b
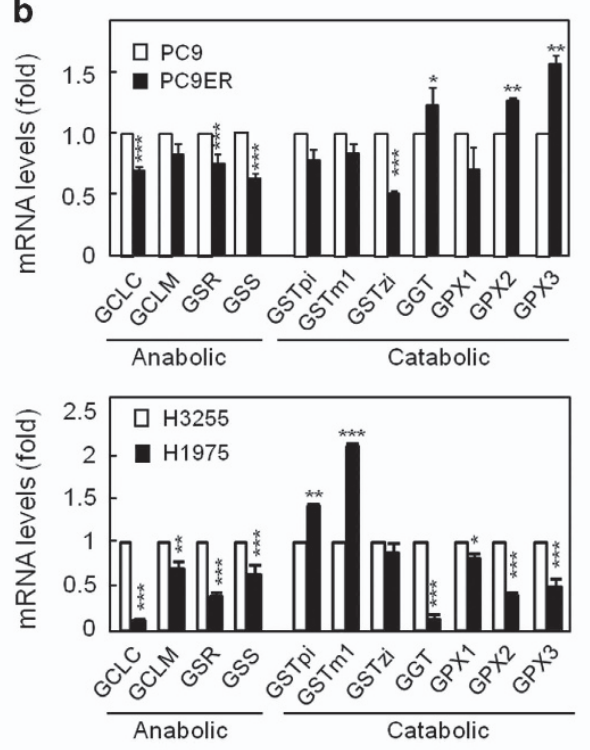
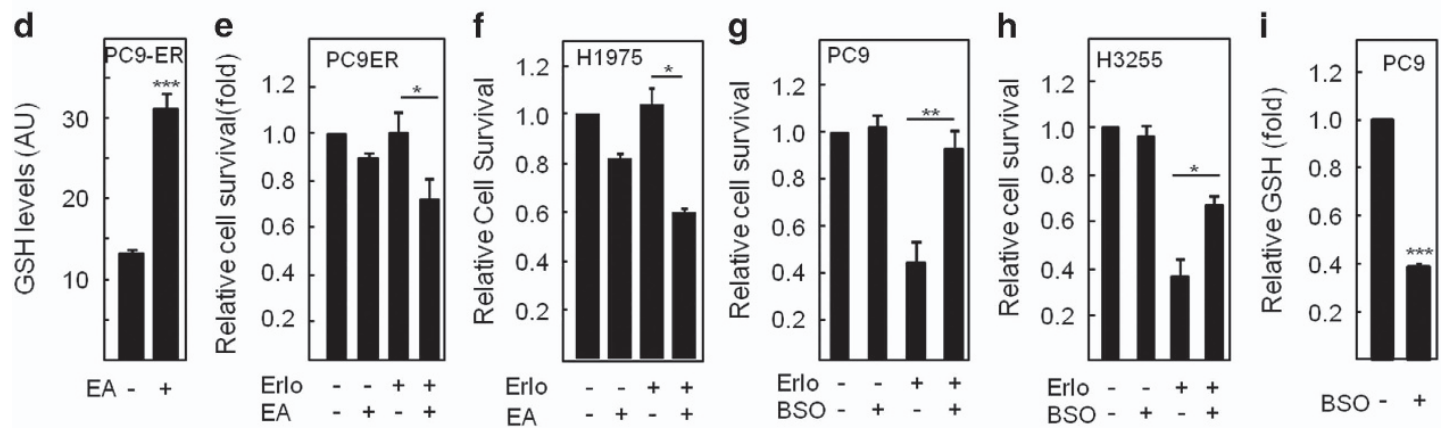

Figure 2 Intracellular GSH levels modulate response to erlotinib. (a) Schematics of the GSH metabolic pathway. White boxes, synthesising enzymes; and grey boxes, catabolic enzymes. (b) Quantitative reverse transcription PCR for GSH pathway enzymes in PC9, PC9ER, H3255 and H1975 cells. Data are relative mRNAs levels in PC9ER (upper panel) and H1975 (lower panel) normalised to those in PC9 and H3255 cells, respectively. (c) PC9 and PC9ER cells were transfected with siRNA targeting GSHcatabolic (grey bars) and synthesising (white bars) enzymes or a non-targeting control (NT) and cell survival to erlotinib (50 nм) monitored by crystal violet staining. Data for the relative survival to erlotinib are normalised to non-targeting control. Survival to erlotinib of PC9ER (e) and H1975 (f) cells treated with ethacrynic acid (EA) or PC9 (g) and H3255 (h) cells treated with buthionine sulphoximine (BSO) was monitored by crystal violet staining. Accompanying changes in GSH levels in PC9ER (d) and PC9 (i) cells were assessed by colorimetric assay. (e-h) Data are the relative responsiveness to erlotinib normalised to vehicle (- ; DMSO). (b-i) Data representative of $\geqslant 3$ experiments and are average of $n=3 \pm$ s.e.m. Statistics: (e-h) analysis of variance, (b-d, i) Student's $t$-test, ${ }^{\star} P \leqslant 0.05,{ }^{* \star} P \leqslant 0.01,{ }^{* *} P \leqslant 0.001$. See also Supplementary Figures S3 and S4.

analysis of the culture medium from our four cell lines disproved this possibility by showing no difference in secreted GSH between TKI-resistant and -sensitive cells (Supplementary Figure S2D). Hence, decreased intracellular GSH levels in erlotinib-resistant cells are likely due to the changes in GSH metabolism.

\section{Erlotinib-resistant cells have lower expression of GSH-synthesising enzymes}

We investigated whether erlotinib-resistant cells differed from their sensitive counterparts in their GSH-metabolic enzymes expression pattern. Quantitative PCR analysis revealed lower messenger
RNA (mRNA) levels for GSH-synthesising enzymes (GCLC, GSS and GSR) in erlotinib-resistant cells compared with sensitive ones (Figure $2 \mathrm{a}$ and $\mathrm{b}$ ). In addition, mRNA levels for GCLM, the modulatory subunit of GCLC, were significantly lower in H1975 than in $\mathrm{H} 3255$ cells. In contrast, changes in the levels for GSH-catabolic enzymes (GPX1/2/3, GGT and GSTpi/m1/zi) varied greatly between cell line pairs and enzyme subtypes indicating no clear pattern (Figure 2b). Therefore, a reduction in GSH biosynthesis becomes a sound explanation for the decreased GSH levels in EGFRm/T790M erlotinibresistant cells. 
Targeting GSH metabolism modulates the cellular response to erlotinib

NMR results suggested that lower GSH levels associated with erlotinib resistance. To strengthen this link, we employed small interfering RNAs (siRNAs) for GSH-metabolic enzymes to modulate GSH levels in our cell lines. Silencing of GSH-catabolic enzymes (GGT1, GPX1 and GSTpi) increased the response
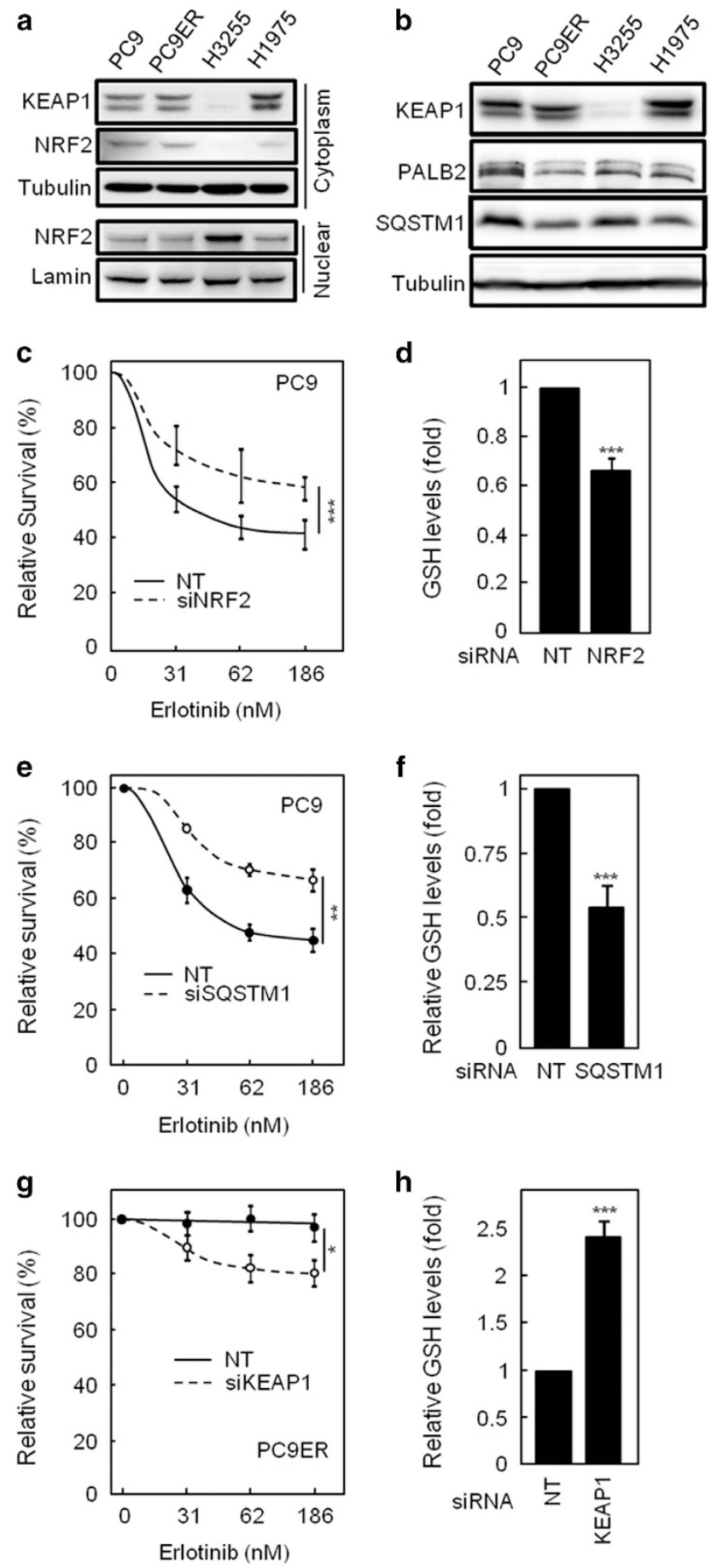

to erlotinib in both the EGFRm PC9 and EGFRm/T790M PC9ER and H1975 cells (Figure 2c; Supplementary Figure S3C). This correlated with efficient targets' downregulation and a corresponding increase in GSH levels (Supplementary Figure S3A and B). Conversely, silencing GSH-synthesising enzymes (GCLC, GSS and GSR) lowered cellular GSH levels (Supplementary Figure S3A and B) and rendered the sensitive PC9 cells erlotinib-resistant (Figure 2c).

To validate our siRNA data, we used smallmolecule inhibitors targeting the activity of GSH pathway enzymes. Treatment with EA, a known GST inhibitor, increased GSH levels in erlotinib-resistant cells (Figure 2d) causing re-sensitisation of PC9ER and H1975 cells to erlotinib (Figure 2e and f). Similarly, GPXs inhibition using mercaptosuccinate increased intracellular GSH levels (Supplementary Figure S3D) and the response of H1975 cells to erlotinib (Supplementary Figure S3E). Conversely, GCLC inhibition using buthionine sulphoximine in sensitive cells made them erlotinib resistant (Figure $2 \mathrm{~g}$ and $\mathrm{h}$ ), an effect associated with decreased GSH levels (Figure 2i). Furthermore, EA was also able to sensitise PC9ER cells to gefitinib by increasing intracellular GSH (Supplementary Figure S4A-C). Taken together, these data suggest that manipulating GSH levels controls the responsiveness of our cell lines to erlotinib.

\section{The NRF2 pathway controls GSH synthesis and responsiveness to erlotinib}

GCLC, GSS and GSR are transcriptional targets of NFE2-related factor 2 (NRF2) [29-31], a downstream target of EGFR [32]. We therefore hypothesised that NRF2 activity might be impaired in EGFRm/T790M cells. NRF2's transcriptional activity requires its nuclear localisation and NRF2 is also degraded through binding to KEAP1, a process counteracted by

Figure 3 Erlotinib resistance correlates with decreased NRF2 activity. (a, b) Subcellular fractions (a) and total lysates (b) from PC9, PC9ER, H3255 and H1975 cells were analysed by SDS-PAGE/western blotting for the indicated proteins. Detection of lamin and tubulin was used as loading controls for nuclear fractions and total lysates or cytoplasmic fractions, respectively. (c-h) PC9 cells transfected with non-targeting (NT), NRF2 or SQSTM1 SiRNAs (c-f) or PC9ER cells transfected with KEAP1 or NT siRNAs $(\mathbf{g}, \mathbf{h})$ were treated with erlotinib and survival assessed by crystal violet staining (c, e, g). GSH levels were measured by colorimetric assay $(\mathbf{d}, \mathbf{f}, \mathbf{h})$. (c-h) Data are average of $n=4 \pm$ s.e.m. Statistics: Student's $t$-test, ${ }^{*} P \leqslant 0.05$, ${ }^{* *} P \leqslant 0.01$, ${ }^{* * *} P \leqslant 0.001$. See also Supplementary Figures S5 and S6. 
competitive interaction of the latter protein with PALB2 and/or SQSTM1. Analysis of nucleocytoplasmic fractions and total lysates from our four cell lines revealed that NRF2 or KEAP1 localisation/ expression had no differences between PC9 and PC9ER cells, whereas H3255 cells showed higher level of nuclear NRF2 than H1975 cells (Figure 3a; Supplementary Figure S5A). This correlated with increased KEAP1 expression in H1975 as compared with H3255 cells (Figure 3a; Supplementary Figure S5B). Although these results alone may explain the difference in GSH pathway enzymes expression between the latter two cell lines, they cannot account for that seen between PC9 and PC9ER cells. However, SQSTM1 was downregulated in both PC9ER and H1975 cells as compared with their erlotinib-sensitive counterparts (Figure 3b; Supplementary Figure S5C), whereas PALB2 levels were lower in PC9ER as compared with PC9 cells (Figure 3b; Supplementary Figure S5D). Furthermore, NRF2 has been shown to be a transcriptional regulator of SQSTM1 and, indeed, mRNA levels of SQSTM1 were found to be significantly lower in both the resistant cell line pair (Supplementary Figure S5E and F). Hence, inhibition of NRF2 activity through various mechanisms may be linked to erlotinib resistance in NSCLC cells.

To test this hypothesis, we silenced NRF2, SQSTM1, PALB2 and KEAP1 in our cells. siRNA-mediated silencing of NRF2 (Supplementary Figure S6A) rendered PC9 cells erlotinib resistant, a change associated with lower intracellular GSH (Figure $3 \mathrm{c}$ and d). Indeed, NRF2-silenced cells showed downregulation of the GSH-synthesising enzymes GCLC and GSR (Supplementary Figure S6B), demonstrating a direct link between NRF2 activity and GSH synthesis. Similarly, SQSTM1 silencing (Supplementary Figure S6C and D) decreased the sensitivity of PC9 cells to erlotinib (Figure 3e) in association with a drop in GSH levels (Figure 3f). Conversely, KEAP1 downregulation (Supplementary Figure S6E) sensitised EGFRm/ T790M PC9ER cells to erlotinib (Figure 3g), accompanied by the increased GSH levels (Figure 3h) and increased transcription of GSH-synthesising enzymes GCLC, GSR and GSS (Supplementary Figure S6F). Finally, despite the changes in PALB2 between PC9ER and PC9 cells (Figure 3b), silencing this protein in PC9 cells failed to induce erlotinib resistance or alter $\mathrm{GSH}$ levels (Supplementary Figure S7A and B). Hence, the modulation of NRF2 activity through KEAP1 and SQSTM1 regulates the sensitivity of NSCLC cells to erlotinib.
Inhibition of NRF2 activity and decreased GSH levels are direct consequences of the T790M mutation

Although lower GSH levels and NRF2 activity were associated with T790M-driven erlotinib resistance in our cell lines, this may still be incidental unless the T790M mutation directly induces these changes. We further expressed the active (L858R) or active/resistant (L858R/T790M) EGFR mutants in HEK293 cells that contain low endogenous EGFR levels (Figure 4a). Unlike expression of the L858R-EGFR, expression of the L858R/T790M double-mutant receptor reduced intracellular GSH levels (Figure 4b). This was associated with reduced PALB2 and SQSTM1 expression (Figure 4c). Conversely, transfection with two independent siRNA sequences previously shown to selectively target T790M-mutant EGFR [33]sensitised PC9ER cells to erlotinib (Figure 4d; Supplementary Figure S7C and D) and increased GSH levels (Figure 4e). The latter correlated with a reversal of changes in the expression pattern of GSH metabolic enzymes observed between PC9 and PC9ER cells (Figure 4f vs Figure 2b) and with increased PALB2, SQSTM1 and NRF2 levels in T790M-silenced cells (Figure 4g). Therefore, lower GSH levels in T790M NSCLC cells are a direct consequence of acquiring this mutation and the accompanying impairment of NRF2 activity.

Decrease in GSH correlates with increased nitric oxide levels

As GSH buffers reactive oxidative species, we investigated whether lower GSH levels in erlotinibresistant cells associated with elevated reactive oxidative species. We performed flow cytometry analysis in the presence of dihydroethidine and DAFFM (4-amino-5-methylamino-2', $7^{\prime}$-difluorofluorescein diacetate) to detect superoxide and nitric oxide (NO) species, respectively. Erlotinib-resistant cells showed an increase in NO species (Figure 5a), although they did not show increased superoxide levels. To assess whether this could influence erlotinib resistance, we first silenced the expression of the three NO synthases, NOS1-3. Although siRNA-mediated downregulation of NOS2 and 3 did not impact on erlotinib resistance (not shown), NOS1 silencing sensitised PC9ER cells to erlotinib (Figure 5b). Next, we quenched cellular NO in erlotinib-resistant cells with the NO-trap carboxyPTIO and revealed that this partially re-sensitised PC9ER cells to erlotinib (Figure 5c). Although these data suggest a role for NO in erlotinib resistance, the levels of changes observed as compared with those seen earlier (Figures 2 and 3) suggest that changes in NO are 

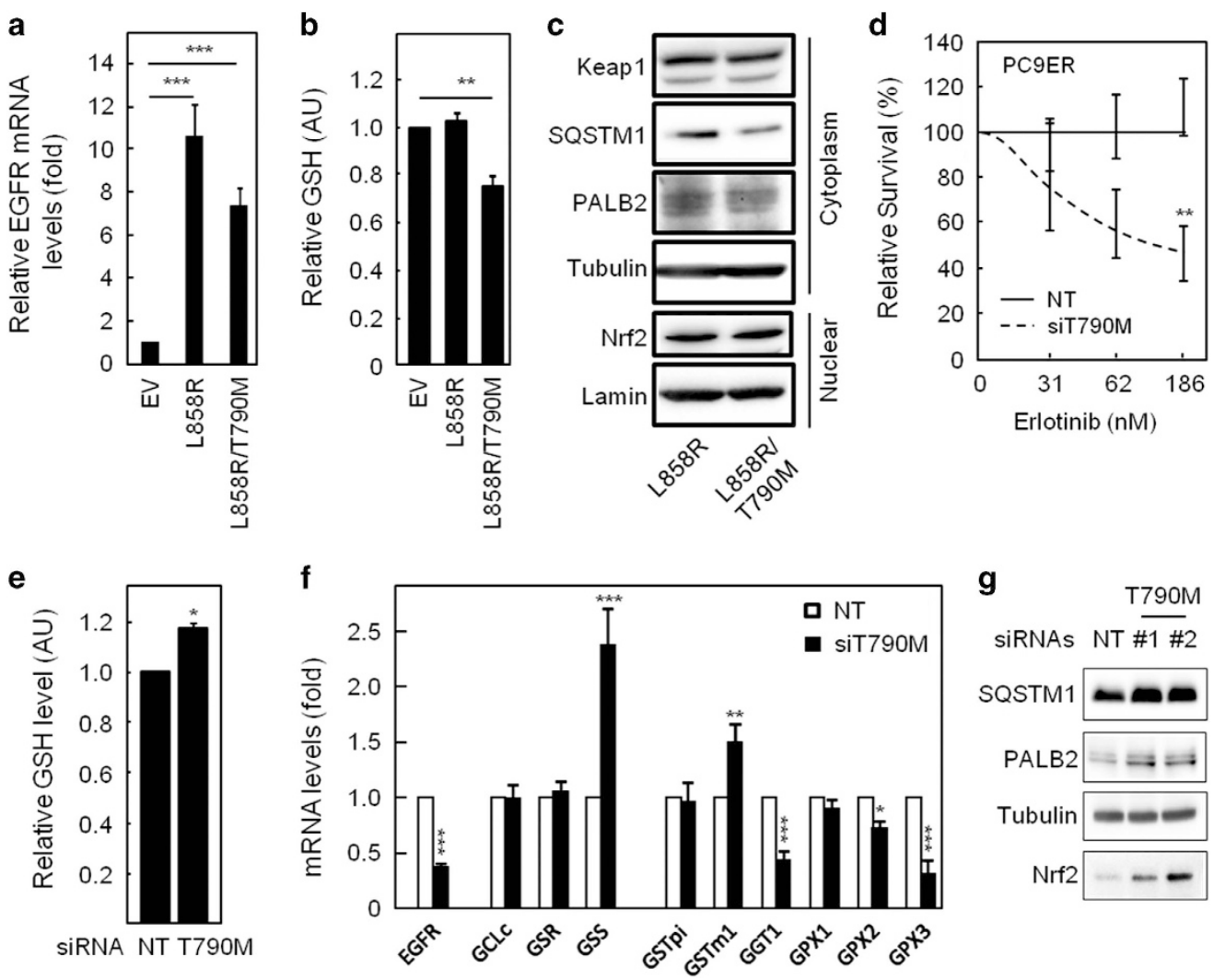

Figure 4 Expression of EGFRm/T790M decreases intracellular GSH levels and NRF2 activity. (a-d) HEK293 cells were transfected with empty vector control (EV), activated L858R-EGFR or activated/resistant L858R/T790M EGFR-mutant constructs. (a) Quantitative reverse transcription PCR for EGFR, (b) colorimetric assay for GSH levels and (c) cell fractionation followed by SDS-PAGE/western blotting for the indicated proteins were done on stable cell lines. Detection of lamin and tubulin was used as loading controls for nuclear and cytoplasmic fractions, respectively. (d-g) PC9ER cells transfected with an EGFR T790M-specific or NT siRNAs were subjected to (d) treatment with erlotinib before crystal violet staining, (e) colorimetric assay for intracellular GSH levels, (f) quantitative PCR for GSH metabolic enzymes or (g) SDS-PAGE/western blotting. All data representative of $\geq 3$ experiments. (a,b,d-f) Values are average of $n=4 \pm$ s.e.m. Statistics: $(\mathbf{a}, \mathbf{b})$ analysis of variance, (d,e,f) Student's $t$-test, ${ }^{*} P \leqslant 0.05,{ }^{* *} P \leqslant 0.01,{ }^{* *} P \leqslant 0.001$. See also Supplementary Figures S6 and S7.

not solely responsible for resistance downstream of decreased GSH levels.

\section{EA administration re-sensitises EGFRm/T790M tumours to erlotinib in mouse xenografts}

The GST inhibitor EA restored GSH levels and erlotinib sensitivity in EGFRm/T790M cells in vitro (Figure 2). EA is still used as a diuretic in humans for conditions including high blood pressure and heart failure [34]. Hence, we hypothesised that co-administration of physiologically relevant doses of EA might improve the responsiveness of EGFRm/ T790M tumours to erlotinib in vivo. PC9 or PC9ER cells were injected subcutaneously in nude mice and tumours were left to grow to $100 \mathrm{~mm}^{3}$. The animals were then treated daily with erlotinib and EA alone or in combination. Co-administration of the drugs greatly inhibited tumour growth with $60 \%$ of the animals showing tumour volumes $\leqslant 300 \mathrm{~mm}^{3}$ at 25 days, whereas those treated with either drug alone showed more extensive disease (Figure 6a). This was associated with increased survival (Figure 6b) and intratumoural GSH levels in combination-treated animals (Figure 6c). EA did not have any effect on erlotinib sensitivity of PC9 xenografts in agreement with the lack of further added sensitisation to erlotinib obtained with this inhibitor in vitro (Supplementary Figure S7E). Thus, co-administration of EA is probably a viable strategy for the management of erlotinib-resistant cancers in humans.

\section{Decreased GSH synthetic enzymes expression characterises erlotinib-resistant patients}

Finally, we assessed whether the decrease in GSH-synthetising enzymes observed in EGFRm/ T790M cell lines in vitro also occurred in patients. 
a
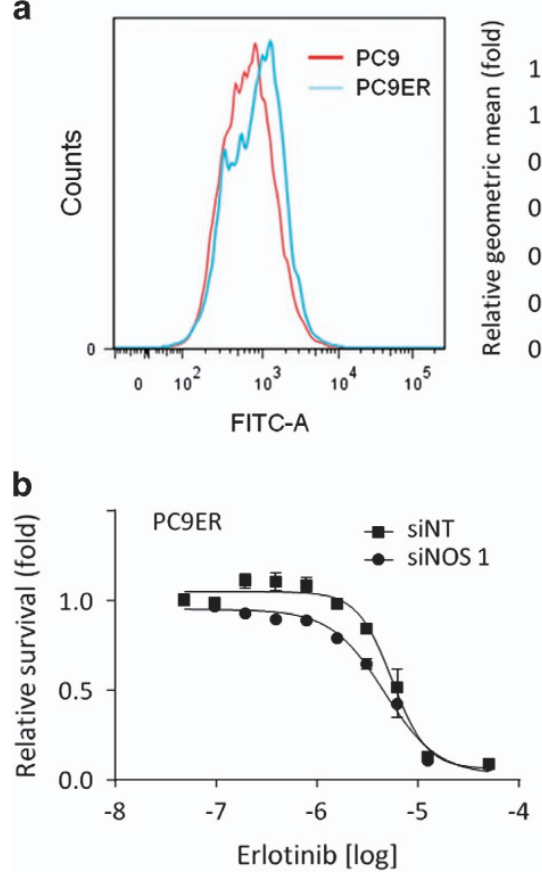

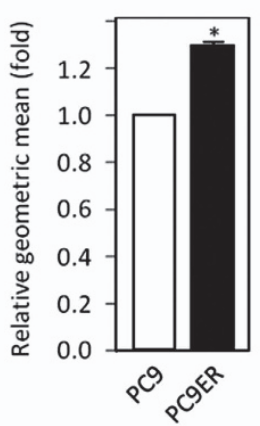

c

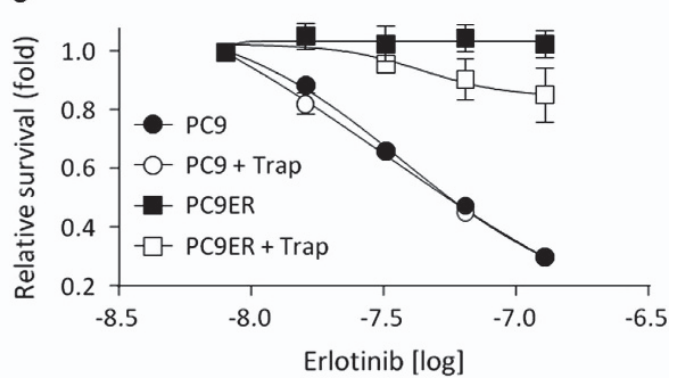

Figure 5 Changes in NO levels modulate erlotinib response. (a) NO levels in PC9 and PC9ER cells were compared by fluorescence-activated cell sorting (FACS) using DAF-FM. Left: FACS profile; right: fold changes in geometric mean. (b) PC9ER cells transfected with non-targeting (NT) or NOS1 siRNAs or (c) PC9 and PC9ER cells treated \pm an NO-trap were exposed to a dose range of erlotinib. Cell survival was determined by crystal violet staining. Statistics: Student's $t$-test, ${ }^{*} P \leqslant 0.05$.

First, we performed quantitative PCR for GSS, GSR, GCLC and GCLM in paired biopsy samples from two patients before (EGFRm alone) and after acquiring EGFRm/T790M-mediated erlotinib resistance. In both cases, resistance was accompanied by a decrease in one or both of the rate-limiting enzymes for GSH biosynthesis, GCLC and GSS (Figure 6d). Moreover, this association was not limited to syngeneic samples, as RNA-Seq of four pairs of unrelated patients' biopsies revealed lower expression of at least one of these enzymes in T790M tumours as compared with non-T790M samples (Figure 6e). Therefore, decreased expression of GSH synthetic enzymes is probably associated with T790M-mediated erlotinib resistance in lung cancer patients.

\section{Discussion}

EGFR TKIs such as erlotinib offer therapeutic benefit to NSCLC patients harbouring EGFRm [1-3]. However, the rapid development of resistance due in $50 \%$ of cases to acquisition of the secondary T790M EGFR mutation greatly limits the ability of these agents to prolong patient survival [5-8]. Although decreased affinity of the EGFRm/T790M for erlotinib was thought responsible and new irreversible inhibitors may be promising in circumventing this, additional mechanisms of resistance are likely to be present. Indeed, EGFRm/T790M cells still demonstrate significant loss of sensitivity to an irreversible compound (Supplementary Figure S1C). This suggested that resistance to erlotinib in EGFRm/T790M NSCLC cells is mediated through additional mechanisms.

Accumulating evidence suggests EGFR mutations to drive alteration in metabolic signatures, however, majority of them fail to demonstrate efficacy of targeting these molecules in clinical settings or in vivo models [35-37]. To identify novel resistance pathways, we performed ${ }^{1} \mathrm{H}-\mathrm{NMR}$ metabonomic analysis of two independent NSCLC erlotinib-sensitive/resistant cell line pairs (PC9/PC9ER and H3255/H1975 cell lines). These were chosen according to the several criteria. First, both resistant cell lines shared the same T790M resistance mutation. Second, although PC9ER cells were obtained through selecting PC9 cells with erlotinib making these two lines relatively isogenic, H3255 and H1975 cells are genetically unrelated. Third, the primary EGFR-activating mutations in the two cell line pairs were different $(\Delta \mathrm{E} 746-\mathrm{A} 750$ for PC9/PC9ER cells, L858R for H3255/H1975 cells). These criteria maximised the opportunity for metabolic changes shared by both cell line pairs to be solely 
a

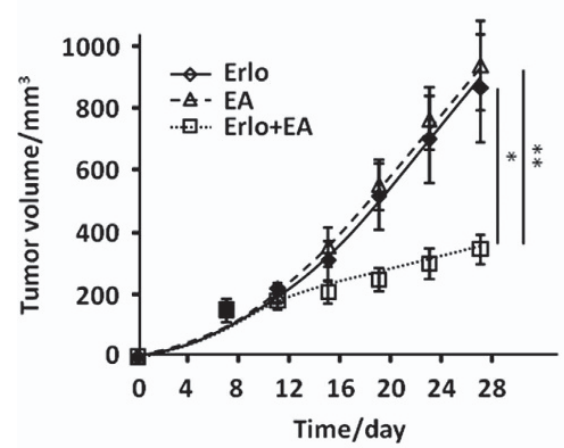

d

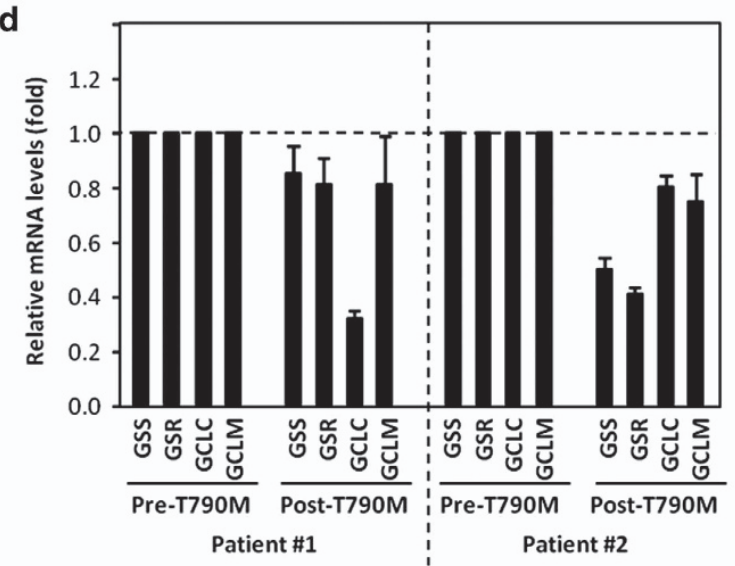

b

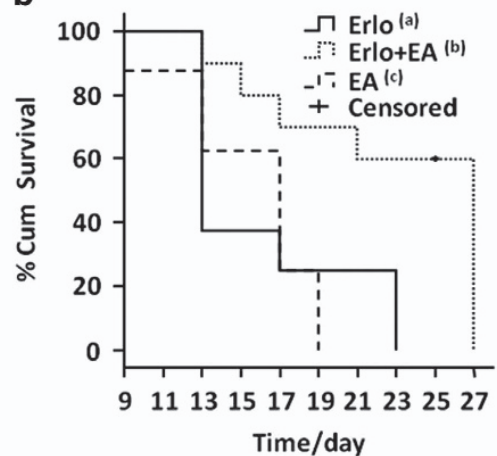

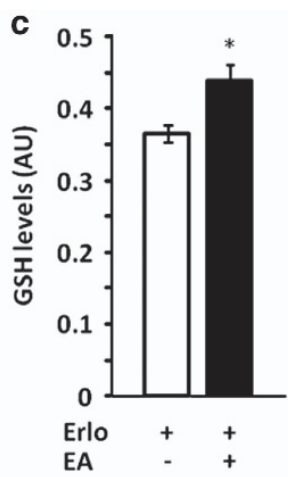

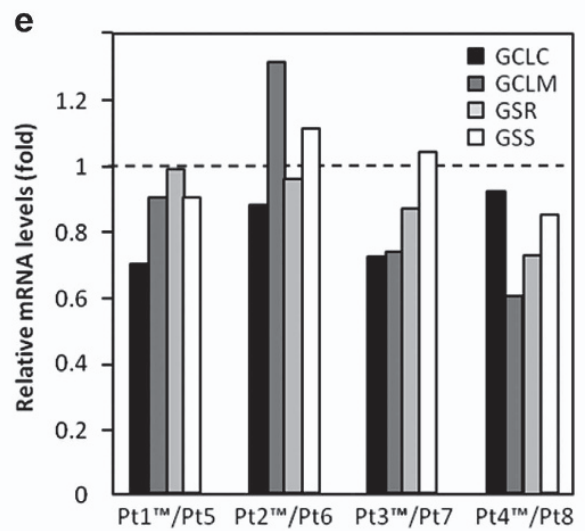

f

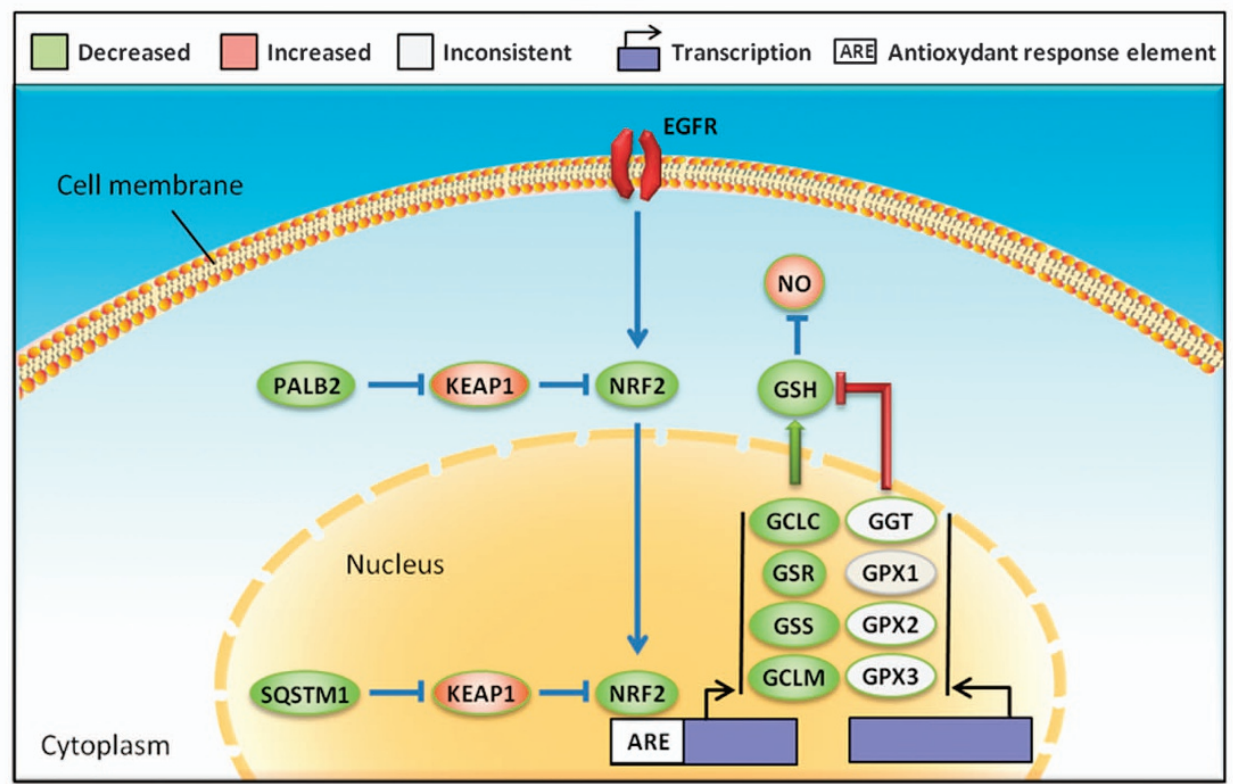

Figure 6 Systemic EA administration re-sensitises PC9ER mouse xenografts to erlotinib. Nude mice ( $n=10 /$ condition) were injected subcutaneously with PC9ER cells and treatment started when tumours reached $100 \mathrm{~mm}^{3}$. (a) Tumour volume and (b) animals survival were monitored for 27 days. (a) Data are average \pm s.e.m. (b) End-point events occur when tumour volumes $\geqslant 300 \mathrm{~mm}^{3}$. Log-rank test, $P_{\mathrm{ab}}<0.01, P_{\mathrm{bc}}<0.01$. (c) Following the last treatment, intratumoral GSH levels were measured ex vivo by colorimetric assay. Statistics: (a) analysis of variance, (c) Student's t-test, ${ }^{*} P<0.05$; ${ }^{* *} P<0.01$. GSH-synthesising enzymes expression is decreased in EGFRm/T790M patient tumours. mRNA levels for the indicated enzymes were compared by quantitative PCR in two patients before (pre-T790M) and after (post-T790M) the onset of T790M-mediated erlotinib resistance (d) or by RNA-Seq in four pairs of unrelated patients with (Pt1-4) or without (Pt5-8) T790M (e). Data in T790M samples are normalised to those in the corresponding non-T790M samples. (f) Model of changes occurring downstream of T790M EGFR. 
dependent on the T790M mutation. One of the most striking differences highlighted by our analysis was a decrease in GSH levels in erlotinib-resistant cells (Figure 1). The GSH pathway has long been involved in cancer drug resistance $[27,28]$. However, this was traditionally associated with increased GSH levels [25, 26]. Indeed, GSH covalently binds to some drug molecules in a GST-dependent manner leading to their cellular export and quenches reactive oxidative species often requiring for these compounds to act $[27,28]$. Therefore, an association between decreased GSH levels and EGFR TKI resistance was surprising and warranted further investigation of its relevance to erlotinib responses.

Our experiments demonstrated that inhibition of GSH biosynthesis by either RNAi or small molecules made erlotinib-sensitive cells resistant to the drug (Figure 2d-i). Conversely, inhibition of GSHdegradation re-sensitised resistant cells to erlotinib (Figure 2d-f). Hence, changes in GSH levels alone can modulate the response of NSCLC cells to this drug and decreased GSH levels accounts for erlotinib resistance in PC9ER and H1975 cells. Comparative analysis revealed a transcriptional downregulation of GSHsynthesising enzymes in T790M cells (Figure 2b) due to the impairment of NRF2, a downstream mediator of EGFR responsible for transcription of these enzymes (Figure 3a and b). This occurred via upregulation of the NRF2 inhibitor KEAP1 and/or downregulation of PALB2 and SQSTM1, two proteins involved NRF2 stabilisation. Indeed, siRNA-mediated silencing of KEAP1 in T790M cells sensitised them to erlotinib (Figure 3g and $\mathrm{h}$ ), whereas that of SQSTM1 or NRF2 made sensitive cells resistant to this drug (Figure 3c-f). Importantly, decreased NRF2 activity and GSH levels in resistant cells were a direct consequence of acquiring the T790M mutation as introducing EGFRm/T790M in HEK293 cells, rather than EGFRm alone, reproduced the changes associated with erlotinib resistance (Figure $4 \mathrm{a}-\mathrm{c}$ ). Conversely, silencing EGFRm/T790M in PC9ER cells reverted the changes in GSH levels and metabolic enzymes seen upon acquisition of resistance by PC9 cells (Figure $4 \mathrm{~d}-\mathrm{g}$ ).

It is unclear by what mechanism(s) the T790M mutation induces the observed transcriptional changes as the higher kinase activity of EGFRm/T790M [9] should further enhance NRF2 activity. However, mutant EGFRs differ from their wild-type counterparts in their subcellular localisation [38], which probably results in the EGFRm/T790M having different signalling partners as EGFRm or wild-type
EGFR. Further research will be required to investigate this possibility.

We next attempted to identify the mechanism by which decreased GSH levels cause erlotinib resistance. GSH is a major cellular antioxidant [39], and its reduced expression could result in increased reactive oxidative species. In addition, single-nucleotide polymorphisms in antioxidant genes have been demonstrated to be associated with survival outcome in patients receiving TKI therapy [40]. Although superoxide levels were unchanged, NO levels were raised in PC9ER as compared with PC9 cells (Figure 5a) and NOS1 silencing or NO quenching sensitised PC9ER cells to erlotinib (Figure $5 \mathrm{~b}$ and c). GSH is known to neutralise $\mathrm{NO}$ and protect against protein nitrosylation $[41,42]$. It is worth noting that EGFR is a target of $S$-nitrosylation [43], but the consequence of this on erlotinib response is currently unknown. However, although our data suggest that NO probably contributes to erlotinib resistance, this does not fully explain the effects of reduced GSH. Glutathionylation has a role in disease state by modifying the function of target proteins [44] and assessing changes to the glutathionylation profile may identify proteins involved in EGFRm/T790M-mediated erlotinib resistance.

Regardless of the mechanism underlying erlotinib resistance downstream of decreased GSH levels, we showed that the GSH pathway could be manipulated for therapeutic benefit. Indeed, systemic administration of clinically relevant doses of EA, a GST inhibitor [45], increased the intra-tumoural GSH levels (Figure 6c) and re-sensitised EGFRm/T790M tumours to erlotinib in a cancer cell xenograft mice model (Figure 6a-c). As EA is an orally available diuretic used in humans with limited toxicity [34], our findings could rapidly translate into clinical practice if this sensitisation also occurs in humans. Moreover, EA has already been used together with classical chemotherapeutics such as alkylating agents to prevent their GST-mediated cellular export [34], leading to improved clinical outcome. Therefore, EA may help manage erlotinib resistance in EGFRm-NSCLC patients and improve response to follow-on chemotherapeutic regimen. However, it is unclear whether decreased GSH levels only occurs downstream of the EGFRm/T790M or if this is a common feature of other erlotinib resistance pathways such as c-Met amplification. Answering this before clinical exploitation of our findings will help more accurate patient selection for EA/erlotinib combined trials.

Finally, we show our findings to be clinically relevant using EGFRm and EGFRm/T790M lung 
cancer samples (Figure 6d and e). The reduced number of samples analysed reflects the fact that repeated biopsy in NSCLC following the onset of EGFR TKI resistance is rare, although this practice is now changing. Nevertheless, we demonstrate in both syngenic and unrelated patient samples that mRNA levels for GSH-synthesising enzymes are decreased in T790M tumours. Hence, probing for GSH-synthesising enzymes may help, in a recurrent setting, to predict the response to combinatorial therapies of erlotinib and GSH level increasing agents.

To sum up, we demonstrate that decreased intracellular level of GSH could mediate T790M-driven erlotinib resistance in NSCLC and highlight the molecular events involved (Figure 6f). Therapeutic strategies that increase intra-tumoural GSH levels may revert erlotinib resistance in the clinic.

\section{Materials and Methods}

\section{Materials}

Mercaptosuccinic acid (used at $50 \mu \mathrm{M}$ ), buthionine sulfoximine (used at $40 \mu \mathrm{M}$ ) and EA (used at 50 and $100 \mu \mathrm{m}$ in PC9 and H1975 cells, respectively) were purchased from Sigma (St Louis, MO, USA), whereas EGFR Inhibitor 324674 was from Santa Cruz (Dallas, TX, USA) and Merck (Kenilworth, NJ, USA), respectively. Antibodies against GSTpi, GPX1, GSS, GSR, GCLC, GSTpi, SQSTM1 and DPP3 were from Abcam (Cambridge, UK); antibodies targeting NRF2 and KEAP1 were from Santa Cruz and anti-PALB2 was from Novus (Abingdon, UK). The specificity of all antibodies employed here was assessed by disappearance of the respective signal following selective targeting of the expression of the corresponding protein by siRNA treatment. Quantitect primers targeting GSTpi, GPX1, GPX1, GSS, GSR and GCLC were from Qiagen (Valencia, CA, USA). All other primers were synthesised by Sigma. SiRNAs were purchased from Dharmacon (Little Chalfont, UK). Dihydroethidine was from Invitrogen (Waltham, MA, USA) and DAF-FM from Sigma.

\section{Cell culture}

All cell lines were obtained from the CRUK cell line bank where they were authenticated and mycoplasma status assessed through regular testing in our lab. Cell lines were grown in Roswell Park Memorial Institute with $10 \%$ fetal bovine serum at $37{ }^{\circ} \mathrm{C}, 5 \% \mathrm{CO}_{2}$.

\section{Extraction of the intracellular metabolites}

Intracellular metabolites were extracted as reported previously $[46,47]$ with some modifications. In brief, $10^{7}$ cells per condition were trypsinised and washed thrice in ice-cold phosphate-buffered saline before metabolite extraction. Cell pellets were resuspended in $0.6 \mathrm{ml}$ cold water/methanol (1:2) and subjected to three freeze-thaw cycles before sonication in a wet ice bath for $15 \mathrm{~min}$ (cycles: $1 \mathrm{~min}$ pulse followed by $1 \mathrm{~min}$ pause). Samples were then centrifuged $\left(3200 \mathrm{~g} / 4^{\circ} \mathrm{C}, 10 \mathrm{~min}\right)$ and supernatants transferred into cold Eppendorfs. The remaining pellets were extracted twice more by the same method. Supernatants from the three subsequent extractions were combined, centrifuged $\left(12000 \mathrm{~g} / 4^{\circ} \mathrm{C}, 10 \mathrm{~min}\right)$ and freeze-dried following vacuum-driven methanol evaporation. Lyophilised samples were stored at $-80^{\circ} \mathrm{C}$. Ten biological replicates were used for each group of cells.

\section{Cellular metabonomic analysis by ${ }^{1} H-N M R$}

Freeze-dried intracellular metabolites extracts were dissolved in $600 \mu$ l phosphate buffer $0.1 \mathrm{~m}\left(\mathrm{pH} 7.4,99.9 \% \mathrm{D}_{2} \mathrm{O}\right)$ containing $0.001 \%$ sodium 3-trimethylsilyl-1-[2,2,3,3- $\left.{ }^{2} \mathrm{H} 4\right]$ propionate as previously described [48]. All samples were centrifuged $\left(12000 \mathrm{~g} / 4^{\circ} \mathrm{C}, 10 \mathrm{~min}\right)$ after short vortexing and supernatants transferred into the $5 \mathrm{~mm}$ NMR tubes for NMR detection. All one-dimensional ${ }^{1} \mathrm{H}$-NMR spectra were acquired on a Bruker AVIII $600 \mathrm{MHz}$ NMR spectrometer equipped with a cryogenic probe (BrukerBiospin, Rheinstetten, Germany) at $298 \mathrm{~K}$. The first increment of NOESY pulse sequence was employed with continuous wave irradiation on the water peak during recycle delay and mixing time for water suppression. Recycle delay of $2 \mathrm{~s}$ and mixing time of $100 \mathrm{~ms}$ were set. The $90^{\circ}$ pulse was adjusted to $10 \mu$ approximately and 64 scans were collected into $32 \mathrm{k}$ data points with the spectral width of 20 p.p.m. For metabolite assignments, two-dimensional NMR spectra including ${ }^{1} \mathrm{H}-{ }^{1} \mathrm{H}$ COSY, ${ }^{1} \mathrm{H}-{ }^{1} \mathrm{H}$ TOCSY, ${ }^{1} \mathrm{H} J$-resolved, ${ }^{1} \mathrm{H}-{ }^{13} \mathrm{C}$ HSQC and ${ }^{1} \mathrm{H}-{ }^{13} \mathrm{C}$ HMBC for typical samples were acquired and processed as described previously [49].

\section{NMR data analysis}

The spectral region at $\delta 0.5-9.5$ was integrated into bins with the width of 0.002 p.p.m. (1.2 Hz) using AMIX package (v3.8, BrukerBiospin). The range ( $\delta$ 4.7-5.2) was removed to eliminate the effects of water peak suppression. Each bin area was normalised to the total area of the respective spectrum. Multivariate data analysis was performed with the software SIMCA-P+ (v 12.0, Umetrics, Umea, Sweden). The model was built using the orthogonal projection to latent structurediscriminant analysis (OPLS-DA) [50] with Pareto variance (Par) scaling and sevenfold cross-validation. The parameter $R^{2} X$ was the variation of $X$ explained by the model and $Q^{2}$ represented the predictability of the model. The validation of all the models was further ensured by $\mathrm{CV}$-analysis of variance $(P<0.05)$ [51]. To assist the biological interpretation of the loadings generated from the models, the loadings was firstly back-transformed [52] and then plotted with colour-coded OPLS-DA coefficients in MATLAB 7.1 using an in-house script [53]. The colour code corresponded to the absolute value of the OPLS-DA coefficients $(|r|)$, indicating the contribution of each variable to explain the intergroup differentiation. The value of $|r|,>0.602$, was considered to be significant $(n=10, P<0.05)$.

\section{GSH colorimetric assay}

A GSH colorimetric assay kit was purchased from BioAssay Systems (Hayward, CA, USA) and used according to the manufacturer's instructions. 


\section{siRNA transfection}

A total of $1 \times 10^{4}$ PC9, PC9ER or H1975 cells per well in six-well plates were transfected with siRNAs at $25 \mathrm{~nm}$ (Dharmacon) for $24 \mathrm{~h}$ using RNAiMax (Invitrogen) following the manufacturer's protocol. Each protein was targeted with a mix of four sequences. A total of $4 \times 10^{3}$ cells were re-seeded and then incubated at $37^{\circ} \mathrm{C} / 5 \% \mathrm{CO}_{2}$ for $24 \mathrm{~h}$ for target silencing before further experiment steps.

\section{Cell survival assay}

For EA, buthionine sulfoximine and mercaptosuccinic acid, cells were pretreated for $4 \mathrm{~h}$ before erlotinib addition (100 nM) for $48 \mathrm{~h}$. Cells were then fixed and stained for $20 \mathrm{~min}$ with a $25 \%$ methanol $/ 0.5 \%$ crystal violet solution. Plates were washed in running water, air-dried and the stain dissolved in $10 \%$ acetic acid on a shaker before absorbance at $595 \mathrm{~nm}$.

\section{Quantitative PCR}

Total cellular mRNA was extracted using the RNeasy kit (Qiagen) and converted to complementary DNA with a High Capacity cDNA Reverse Transcription Kit (Applied Biosystems, Waltham, MA, USA). mRNA levels were quantified using a Fast SYBR Green Master Mix (Applied Biosystems) on a 7900HT Fast Real time PCR System (Applied Biosystems). qBase software (San Francisco, CA, USA) was used for data analysis using TATA-binding protein and $\beta$-actin as internal controls. The primers used were listed below (F, forward; R, reverse): GCLm: (F): 5'-GGCACAGGTAAAACCAAATA GTAAC-3', (R): 5'-CAAATTGTTTAGCAAATGCAGTCA -3'; GPX2: (F): 5'-TAAGTGGGCTCAGGCCTCTCT-3', (R): 5'-GGTCATAGAAGGACTTGGCAATG-3'; GPX3: (F): 5'-GACAAGAGAAGTCGAAGATG-3'，(R): 5'-CTTCCTG TAGTGCATTCAGTT-3'; GSTz1: (F): 5'-TCCTATTTCCG AAGCTCCTGC-3', (R): 5'-TTCAGTGCCTGGAAGTCCTT AG-3'; GSTm1: (F): 5'-CTATGATGTCCTTGACCTCCA CCGTATA-3', (R): 5'-ATGTTCACGAAGGATAGTGGG TAGCTGA-3'; Beta-Actin: (F): 5'-TCCTCCTGAGCGCAA GTACTC-3', (R): 5'-CTGCTTGCTGATCCACATCTG-3'; KEAP1: (F): 5'-CAGATTGGCTGTGTGGAGTT-3'， (R): 5'-GCTGTTCGCAGTCGTACTTG-3'; SQSTM1: (F): 5'-CTGGGACTGAGAAGGCTCAC-3', (R): 5'-GCAGCTG ATGGTTTGGAAAT-3'; TBP: (F): 5'-TGCACAGGAGCCA AGAGTGAA-3', (R): 5'-CACATCACAGCTCCCCACCA-3'; NRF2 primers: (F): 5'-GAGAGCCCAGTCTTCATTGC-3', (R): 5'-TGCTCAATGTCCTGTTGCAT-3'. Primers against the other targets were purchased from Qiagen: GCLc (QT00037310), GGT1 (QT00029470), GPX1 (QT00203392), GSTp1 (QT00086401), GSS (QT00014413) and GSR (QT00038325).

\section{Tissue $m R N A$ extraction and quantitative $P C R$}

The origin of tissues and techniques used are as previously reported [54]. In short, samples were obtained from EGFRmutant lung adenocarcinoma patients with acquired erlotinib resistance under Human Investigations Protocol \#111000928 (Yale Cancer Center, New Haven, CT). Those were reviewed by a pathologist to ensure adequate tumour content. Tumour areas were circled and microdissection performed to enrich for tumour content.

\section{Tissue $m R N A$ extraction and $R N A-S e q$}

The Illumina TruSeq RNA Sample Preparation Kit (Illumina Cambridge Ltd, Essex, UK) was used for RNA tissue extraction and analysis done as previously described [55].

\section{Western blotting}

Cells were lysed using $0.5 \%$ Triton X-100, $150 \mathrm{~mm} \mathrm{NaCl}$, $2 \mathrm{~mm}$ EDTA, $10 \%$ glycerol supplemented with protease inhibitors cocktail tablets (Roche Diagnostics, Indianapolis, IN, USA), $10 \mathrm{~mm} \beta$-glycerophosphate, $1 \mathrm{~mm} \mathrm{Na}_{3} \mathrm{VO}_{4}$ and $10 \mathrm{~mm}$ $\mathrm{NaF}$. Equal protein amounts were analysed by SDS-PAGE/ western blotting using the antibodies indicated.

\section{Flow cytometry analysis of oxidative species}

Cells $\left(15 \times 10^{4} /\right.$ well in a six-well plate) were treated with $10 \mu \mathrm{M}$ DAF-FM or dihydroethidine for $30 \mathrm{~min}$, washed with phosphate-buffered saline, trypsinised, pelleted and resuspended in $1 \mathrm{ml}$ of phosphate-buffered saline before flow cytometry using a BD FACSCalibur (DB Biosciences, Oxford, UK). The geometric mean intensity was determined using FlowJo (Tree Star Inc, Ashland, OR, USA).

\section{Animal experiments}

A total of $5 \times 10^{6}$ PC9ER or PC9 cells were injected subcutaneously into the flank of female BALB/c nude mice and the tumours grew until they reached $100 \mathrm{~mm}^{3}$. Mice were then randomized into three groups $(n=10)$ and treated by intraperitoneal injection of $25 \mathrm{mg} \mathrm{kg}^{-1}$ per day erlotinib/0.5\% w/v methylcellulose and/or $6 \mathrm{mg} \mathrm{kg}^{-1}$ per day EA/1\% Tween 80 in distilled water. Such treatments were administered daily from day 7 to 26 . Tumours were measured by caliper and volumes calculated as $V=1 / 2 * L^{*} W^{2}$ ( $L$; length, $W$; width of tumour). Data analysis was performed by an investigator blinded to the experimental conditions. All experiments complied with ethical regulations as enforced by the local committee.

\section{Conflict of Interest}

The authors declare no conflict of interest.

\section{Acknowledgements}

HRT/YLW acknowledges the financial supports from the Ministry of Science and Technology of China (2012CB934004), National Natural Science Foundation of China (81590953, 81590950, 91439102 and 21375144). MJS/OP is funded by the European Commission's FP7 under the LungTarget consortium, the AICR and CTRT. Also, MJS is supported by CRUK and UK Department of Health/NIHR Imperial ECMC and BRC.

\section{Author contributions}

MJS, OEP, YLW and HRT conceived and designed the study. HDL did the metabolomics data acquisition and analysis. HDL, YLH and ZGL conducted all the animal experiments and data analysis. WS, EC and RR did the molecular biology data 
acquisition and analysis. EB, EFS, GJJEH and JD acquired the mRNA data on gene expression in clinical samples. OEP, YLW, MJS and HRT did the overall data interpretation with others and wrote the paper.

\section{References}

1 Mok TS, Wu YL, Yu CJ et al. Randomized, placebocontrolled, phase II study of sequential erlotinib and chemotherapy as first-line treatment for advanced nonsmall-cell lung cancer. J Clin Oncol 2009; 27: 5080-5087.

2 Lynch TJ, Bell DW, Sordella R et al. Activating mutations in the epidermal growth factor receptor underlying responsiveness of non-small-cell lung cancer to gefitinib. N Engl J Med 2004; 350: 2129-2139.

3 Paez JG, Janne PA, Lee JC et al. EGFR mutations in lung cancer: correlation with clinical response to gefitinib therapy. Science 2004; 304: 1497-1500.

4 Stewart EL, Tan SZ, Liu G, Tsao MS. Known and putative mechanisms of resistance to EGFR targeted therapies in NSCLC patients with EGFR mutations-a review. Transl Lung Cancer Res 2015; 4: 67-81.

5 Kosaka T, Yatabe Y, Endoh H et al. Analysis of epidermal growth factor receptor gene mutation in patients with non-small cell lung cancer and acquired resistance to gefitinib. Clin Cancer Res 2006; 12: 5764-5769.

6 Balak MN, Gong Y, Riely GJ et al. Novel D761Y and common secondary T790M mutations in epidermal growth factor receptor-mutant lung adenocarcinomas with acquired resistance to kinase inhibitors. Clin Cancer Res 2006; 12: 6494-6501.

7 Kobayashi $\mathrm{S}$, Boggon TJ, Dayaram $\mathrm{T}$ et al. EGFR mutation and resistance of non-small-cell lung cancer to gefitinib. N Engl J Med 2005; 352: 786-792.

8 Pao W, Miller VA, Politi KA et al. Acquired resistance of lung adenocarcinomas to gefitinib or erlotinib is associated with a second mutation in the EGFR kinase domain. PLoS Med 2005; 2: e73.

9 Yoshikawa S, Kukimoto-Niino M, Parker L et al. Structural basis for the altered drug sensitivities of nonsmall cell lung cancer-associated mutants of human epidermal growth factor receptor. Oncogene 2012; 32: 27-38.

10 Cross DA, Ashton SE, Ghiorghiu S et al. AZD9291, an irreversible EGFR TKI, overcomes T790M-mediated resistance to EGFR inhibitors in lung cancer. Cancer Discov 2014; 4: 1046-1061.

11 Walter AO, Sjin RT, Haringsma HJ et al. Discovery of a mutant-selective covalent inhibitor of EGFR that overcomes T790M-mediated resistance in NSCLC. Cancer Discov 2013; 3: 1404-1415.

12 Zhang Q, Liu Y, Gao F et al. Discovery of EGFR selective 4,6-disubstituted pyrimidines from a combinatorial kinase-directed heterocycle library. J Am Chem Soc 2006; 128: 2182-2183.

13 Miller VA, Hirsh V, Cadranel J et al. Afatinib versus placebo for patients with advanced, metastatic non-smallcell lung cancer after failure of erlotinib, gefitinib, or both, and one or two lines of chemotherapy (LUX-Lung 1): a phase 2b/3 randomised trial. Lancet Oncol 2012; 13: 528-538.

14 Costa DB, Kobayashi SS. Whacking a mole-cule: clinical activity and mechanisms of resistance to third generation EGFR inhibitors in EGFR mutated lung cancers with EGFR-T790M. Transl Lung Cancer Res 2015; 4: 809-815.

15 Jones RG, Thompson CB. Tumor suppressors and cell metabolism: a recipe for cancer growth. Genes Dev 2009; 23: $537-548$.

16 Cavill R, Kamburov A, Ellis JK et al. Consensusphenotype integration of transcriptomic and metabolomic data implies a role for metabolism in the chemosensitivity of tumour cells. PLoS Comput Biol 2011; 7: e1001113.

17 Jang M, Kim SS, Lee J. Cancer cell metabolism: implications for therapeutic targets. Exp Mol Med 2013; 45: e45.

18 Zhao Y, Butler EB, Tan M. Targeting cellular metabolism to improve cancer therapeutics. Cell Death Dis 2013; 4: e532.

19 Sreekumar A, Poisson LM, Rajendiran TM et al. Metabolomic profiles delineate potential role for sarcosine in prostate cancer progression. Nature 2009; 457: 910-914.

20 Boros LG, Cascante M, Lee WN. Metabolic profiling of cell growth and death in cancer: applications in drug discovery. Drug Discov Today 2002; 7: 364-372.

21 Wang YH, Zhang LM, Chen WL et al. Rapid diagnosis and prognosis of de novo acute myeloid leukemia by serum metabonomic analysis. J Proteome Res 2013; 12: 4393-4401.

22 Serkova NJ, Spratlin JL, Eckhardt SG. NMR-based metabolomics: translational application and treatment of cancer. Curr Opin Mol Ther 2007; 9: 572-585.

23 Tian Y, Nie X, Xu S et al. Integrative metabonomics as potential method for diagnosis of thyroid malignancy. Sci Rep 2015; 5: 14869.

24 Alama A, Orengo AM, Ferrini S, Gangemi R. Targeting cancer-initiating cell drug-resistance: a roadmap to a new-generation of cancer therapies? Drug Discov Today 2012; 17: 435-442.

25 Brozovic A, Ambriovic-Ristov A, Osmak M. The relationship between cisplatin-induced reactive oxygen species, glutathione, and BCL-2 and resistance to cisplatin. Crit Rev Toxicol 2010; 40: 347-359.

26 Bracht K, Boubakari, Grunert R, Bednarski PJ. Correlations between the activities of 19 anti-tumor agents and the intracellular glutathione concentrations in a panel of 14 human cancer cell lines: comparisons with the National Cancer Institute data. Anticancer Drugs 2006; 17: 41-51.

27 Provost K, Bouvet-Muller D, Crauste-Manciet S et al. EXAFS structural study of platinum-based anticancer drugs degradation in presence of sulfur nucleophilic species. Biochimie 2009; 91: 1301-1306.

28 Rappa G, Lorico A, Flavell RA, Sartorelli AC. Evidence that the multidrug resistance protein (MRP) functions as a co-transporter of glutathione and natural product toxins. Cancer Res 1997; 57: 5232-5237.

29 Bell KF, Fowler JH, Al-Mubarak B, Horsburgh K, Hardingham GE. Activation of Nrf2-regulated glutathione pathway genes by ischemic preconditioning. Oxid Med Cell Longev 2011; 2011: 689524. 
30 Kato K, Takahashi K, Monzen S et al. Relationship between radiosensitivity and Nrf2 target gene expression in human hematopoietic stem cells. Radiat Res 2010; 174: 177-184.

31 Sekhar KR, Crooks PA, Sonar VN et al. NADPH oxidase activity is essential for Keap1/Nrf2-mediated induction of GCLC in response to 2-indol-3-yl-methylenequinuclidin-3ols. Cancer Res 2003; 63: 5636-5645.

32 Papaiahgari S, Zhang Q, Kleeberger SR, Cho HY, Reddy SP. Hyperoxia stimulates an Nrf2-ARE transcriptional response via ROS-EGFR-PI3K-Akt/ERK MAP kinase signaling in pulmonary epithelial cells. Antioxid Redox Signal 2006; 8: 43-52.

33 Chen G, Kronenberger P, Teugels E, Umelo IA, De Greve J. Effect of siRNAs targeting the EGFR T790M mutation in a non-small cell lung cancer cell line resistant to EGFR tyrosine kinase inhibitors and combination with various agents. Biochem Biophys Res Commun 2013; 431: 623-629.

34 Somberg JC, Molnar J. The pleiotropic effects of ethacrynic acid. Am J Ther 2009; 16: 102-104.

35 Makinoshima $\mathrm{H}$, Takita $\mathrm{M}$, Saruwatari $\mathrm{K}$ et al. Signaling through the phosphatidylinositol 3-kinase (pi3k)/ mammalian target of rapamycin (mtor) axis is responsible for aerobic glycolysis mediated by glucose transporter in epidermal growth factor receptor (egfr)-mutated lung adenocarcinoma. J Biol Chem 2015; 290: 17495-17504.

36 Serizawa M, Kusuhara M, Zangiacomi V et al. Identification of metabolic signatures associated with erlotinib resistance of non-small cell lung cancer cells. Anticancer Res 2014; 34: 2779-2787.

37 Xie C, Jin J, Bao X et al. Inhibition of mitochondrial glutaminase activity reverses acquired erlotinib resistance in non-small cell lung cancer. Oncotarget 2016; 7: 610-621.

38 Chung BM, Raja SM, Clubb RJ et al. Aberrant trafficking of NSCLC-associated EGFR mutants through the endocytic recycling pathway promotes interaction with Src. BMC Cell Biol 2009; 10: 84.

39 Schafer FQ, Buettner GR. Redox environment of the cell as viewed through the redox state of the glutathione disulfide/glutathione couple. Free Radic Biol Med 2001; 30: 1191-1212.

$40 \mathrm{Xu} \mathrm{Y,} \mathrm{Pan} \mathrm{Q,} \mathrm{Wang} \mathrm{C} \mathrm{et} \mathrm{al.} \mathrm{Genetic} \mathrm{polymorphisms} \mathrm{in}$ oxidative stress-related genes are associated with clinical outcome in patients with advanced non-small cell lung cancer receiving tyrosine kinase inhibitors. Am J Cancer Res 2014; 4: 934-942.

41 Pedersen JZ, De Maria F, Turella P et al. Glutathione transferases sequester toxic dinitrosyl-iron complexes in cells. A protection mechanism against excess nitric oxide. J Biol Chem 2007; 282: 6364-6371.

42 Aquilano K, Baldelli S, Ciriolo MR. Glutathione is a crucial guardian of protein integrity in the brain upon nitric oxide imbalance. Commun Integr Biol 2011; 4: 477-479.

43 Switzer CH, Glynn SA, Cheng RY et al. S-nitrosylation of EGFR and Src activates an oncogenic signaling network in human basal-like breast cancer. Mol Cancer Res 2012; 10: 1203-1215.

44 Ghezzi P. Protein glutathionylation in health and disease. Biochim Biophys Acta 2013; 1830: 3165-3172.

45 Ploemen JH, van Ommen B, Bogaards JJ, van Bladeren PJ. Ethacrynic acid and its glutathione conjugate as inhibitors of glutathione S-transferases. Xenobiotica 1993; 23: 913-923.

46 Li HD, Zhu WD, Zhang LK et al. The metabolic responses to hepatitis B virus infection shed new light on pathogenesis and targets for treatment. Sci Rep 2015; 5: 8421.

47 Zhang LM, Wang LM, Hu YL et al. Selective metabolic effects of gold nanorods on normal and cancer cells and their application in anticancer drug screening. Biomaterials 2013; 34: 7117-7126.

48 Jiang LM, Huang J, Wang YL, Tang HR. Eliminating the dication-induced intersample chemical-shift variations for NMR-based biofluid metabonomic analysis. Analyst 2012; 137: 4209-4219.

49 An YP, Xu WX, Li HH et al. High-fat diet induces dynamic metabolic alterations in multiple biological matrices of rats. J Proteome Res 2013; 12: 3755-3768.

50 Trygg J, Wold S. Orthogonal projections to latent structures (O-PLS). J Chemom 2002; 16: 119-128.

51 Eriksson L, Trygg J, Wold S. CV-ANOVA for significance testing of PLS and OPLS (R) models. J Chemom 2008; 22: 594-600.

52 Cloarec O, Dumas ME, Craig A et al. Statistical total correlation spectroscopy: an exploratory approach for latent biomarker identification from metabolic $1 \mathrm{H}$ NMR data sets. Anal Chem 2005; 77: 1282-1289.

53 Li D, Zhang LL, Dong FC et al. Metabonomic changes associated with atherosclerosis progression for LDLR-/mice. J Proteome Res 2015; 14: 2237-2254.

54 deBruin EC, Cowell C, Warne PH et al. Reduced NF1 expression confers resistance to EGFR inhibition in lung cancer. Cancer Discov 2014; 4: 606-619.

55 Huang S, Holzel M, Knijnenburg $\mathrm{T}$ et al. MED12 controls the response to multiple cancer drugs through regulation of TGF-beta receptor signaling. Cell 2012; 151: 937-950.

(Supplementary information is linked to the online version of the paper on the Cell Discovery website.)

This work is licensed under a Creative Commons Attribution 4.0 International License. The images or other third party material in this article are included in the article's Creative Commons license, unless indicated otherwise in the credit line; if the material is not included under the Creative Commons license, users will need to obtain permission from the license holder to reproduce the material. To view a copy of this license, visit http://creativecommons.org/licenses/by/4.0/

(C) The Author(s) 2016 